\title{
Hybrid Modeling of Flotation Height in Air Flotation Oven Based on Selective Bagging Ensemble Method
}

Shuai Hou, Fuan Hua, Wu Lv, Zhaodong Wang, Yujia Liu, and Guodong Wang

The State Key Laboratory of Rolling and Automation, Northeastern University, Shenyang 110819, China

Correspondence should be addressed to Shuai Hou; houshuai20072@163.com

Received 20 September 2013; Accepted 14 November 2013

Academic Editor: Didier Georges

Copyright ( 2013 Shuai Hou et al. This is an open access article distributed under the Creative Commons Attribution License, which permits unrestricted use, distribution, and reproduction in any medium, provided the original work is properly cited.

\begin{abstract}
The accurate prediction of the flotation height is very necessary for the precise control of the air flotation oven process, therefore, avoiding the scratch and improving production quality. In this paper, a hybrid flotation height prediction model is developed. Firstly, a simplified mechanism model is introduced for capturing the main dynamic behavior of the process. Thereafter, for compensation of the modeling errors existing between actual system and mechanism model, an error compensation model which is established based on the proposed selective bagging ensemble method is proposed for boosting prediction accuracy. In the framework of the selective bagging ensemble method, negative correlation learning and genetic algorithm are imposed on bagging ensemble method for promoting cooperation property between based learners. As a result, a subset of base learners can be selected from the original bagging ensemble for composing a selective bagging ensemble which can outperform the original one in prediction accuracy with a compact ensemble size. Simulation results indicate that the proposed hybrid model has a better prediction performance in flotation height than other algorithms' performance.
\end{abstract}

\section{Introduction}

Air flotation oven is a type of advanced heat treatment equipment. By virtue of the air flotation ovens, a variety of strips with high surface quality and high performance can be obtained [1]. Due to its excellent performance, a considerable attention and many excellent researches on this topic have been reported in the literature [1-9]. In air flotation oven, the flotation height of the strip is an important factor. However, the flotation height is difficult be measure because of high-temperature work environment and signal interference, which is an obstacle to the optimal control of the process and may reduce the product quality. Therefore, the research on the prediction of flotation height becomes more and more attractive in the air flotation oven $[1,5,9]$ based on which high-precision control can be realized. As a result, highproduct quality can be finally obtained.

Mechanism modeling based on fluid mechanics and solid mechanics is a way for establishing the prediction model of flotation height. There are some mechanism models that can be found in the literature [1-8]. Green's function and
Galerkin's method are applied to the research of air flotation oven. The theoretical calculation and experiment are reported. The flotation height of strip is predicted in theory calculation and compared with experiment result [1]. The basic theory governing of air flotation oven is discussed which has been used to predict the flotation height of air cushion craft [3]. The flotation height is given which is based on the extensional resiliency model of an air-floated web [5]. The formula of flotation height is given which is based on the strip's lateral deflection. The governing partial differential equations are applied to the lateral deflection of strip [6]. In summary, the mechanism models are good tools for process analysis. However, there are some drawbacks. One major drawback is that the mechanism model should be based on strict assumptions, such as linearity assumption and independence assumption among variables. As a result, the prediction accuracy of the mechanism model will be decreased. Furthermore, the mechanism models generally have a complex structure which makes them difficult to adjust. Moreover, the mechanism model may involve partial-differential part or integral part that is hard to solve and the computational cost is 
considerable, which makes it unsuitable for online industrial control. The above drawbacks limit the application of mechanism method to the real world and bring a low prediction accuracy in flotation height prediction.

In the past decades, process modeling by machine learning algorithms has drawn more and more attention and has been applied to the industrial process successfully [8-19]. Various machine learning algorithms have the advantages of high accuracy and simple modeling process. The machine learning process can automatically extract knowledge from training data, by which the difficult-to-measure variable flotation height can be predicted by the easy-to-measure variables. According to previous studies, machine learning can learn the complex process or nonlinear relationship between input-output variables very well. Finally, a simple structure model can be derived [11]. Neural network and SVM are two popular machine learning algorithms $[12,13]$. They possess good learning ability and have been widely used in various process modeling problems. However, neural network and SVM have their own drawbacks. There are some parameters in these learning algorithms which are hardly determined. Moreover, these learning algorithms easily overfit the training data. As a result, the prediction performance becomes bad. For solving these problems, ensemble learning has been proposed recently [20-24]. Ensemble learning constructs a highly accurate prediction model by combining an ensemble of several neural network, or SVMs. The individual NN or SVM in the ensemble needs only to be moderately accurate on the training set. Many research studies prove that the ensemble model shows better prediction performance compared with the individual models.

Ensemble learning has drawn many researchers' attention in the literature recently [20-27]. Bagging is a famous ensemble learning algorithm which has already been widely used to improve the accuracy of classification and regression problems [21-24]. The advantage of bagging is the good performance of robustness $[21,22]$. The disadvantage of bagging is that the individual models are not cooperated with each other, which may establish a relatively large ensemble size and low accurate ensemble learning model. NCL is another famous ensemble learning method [25-27], which explicitly promotes cooperation between individual models. Therefore, its learning ability is perfect. A drawback of NCL is that the overfitting problem may occur. In summary, the common problems of various ensemble learning algorithms are the determination of the optimal ensemble size, the training of the base learners, and the fusion strategy of the ensemble.

In this paper, a hybrid flotation height prediction model is developed for combining the well generalization performance of mechanism modeling method and the excellent learning ability of machine learning algorithms. In the framework of the proposed hybrid model, a simplified mechanism model is introduced for description of the main knowledge and complemented by an error compensation model in the air flotation process. The simplified mechanism model is based on thin jet model which is a branch of fluid mechanics and is very suitable for description of the behavior in air flotation oven. Furthermore, in order to compensate the modeling error of the simplified mechanism model and improve the flotation height prediction accuracy, an error compensation model is introduced for describing the unknown structure part that is hardly modeled by the mechanistic way. Because of the excellent ability of machine learning in nonlinearity problem and complex process problem the error compensation model is established on the basis of machine learning algorithms. In the current study, an error compensation model is proposed, which is a modification algorithm based on existing ensemble learning algorithms. The proposed ensemble method is basically a selective bagging ensemble method, where GA, NCL, and bagging are combined in the way that the base learners are selected from original bagging ensemble by GA and NCL. The proposed method can retain the robustness property of bagging whilst further improving its prediction accuracy by the well learning ability of NCL method.

The remainder of this paper is organized as follows. In Section 2, the details of the ground effect theories and the mathematical mechanism model of floatation height are presented. In Section 3, the proposed selective bagging ensemble method (SBE) is introduced. Section 4 reports the hybrid model based on mechanism model and selective bagging ensemble model. Section 5 reports the experimental results. Section 6 draws conclusions and future research directions.

\section{Mathematical Mechanism Model of the Flotation Height}

2.1. Brief Review of Air Flotation Process. High quality production of cold rolled metal alloy strips and coating metal strips requires continuous heat treatment including aluminum strip, copper strip, and steel strip. Air flotation ovens are used for effectively coating and heating strips where the metal strip can suspend in the air without contacting anything. Therefore, coating destruction is avoided and good surface quality can be finally realized. Furthermore, it can provide the necessary temperature uniformity along the width and length of strip. As a result, compared with conventional continuous furnaces, air flotation oven can product better performance and quality product in the heat treatment process of cold-rolled metal strip. Commonly, air flotation oven is followed by a sufficiently fast cooling equipment for guaranteeing desired material properties, such as hardness and grain size.

This paper studies an air flotation oven that is specifically used for the heat treatment of aluminum strip, which is schematically shown in Figure 1. The aluminum strips pass through between the upper nozzles and lower nozzles at a constant speed. The aluminum strip is suspended and heated by the hot air emerging from the upper nozzles and lower nozzles which are arranged on upper surface and lower surface of air flotation oven.

It can be seen from Figure 1 that there are two slit nozzles on top surface of the upper nozzles and lower nozzle in parallel. The air is ejected from these slit nozzles and squirted onto the surface of the aluminum strip. The external wall which is parallel to the slit has the angle against the air flotation oven. The ratio of the flow rate of the upper nozzle 


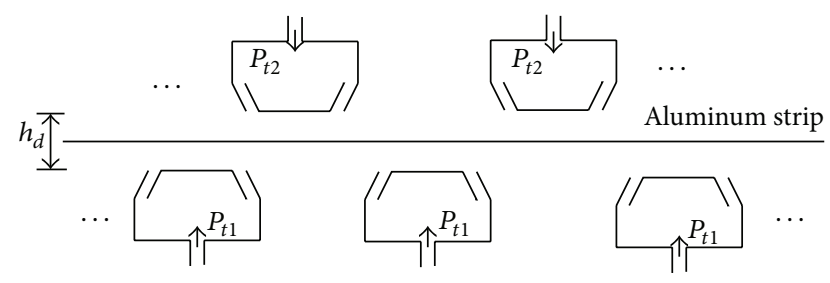

FIgURE 1: Aluminum strip and air bars in air flotation oven.

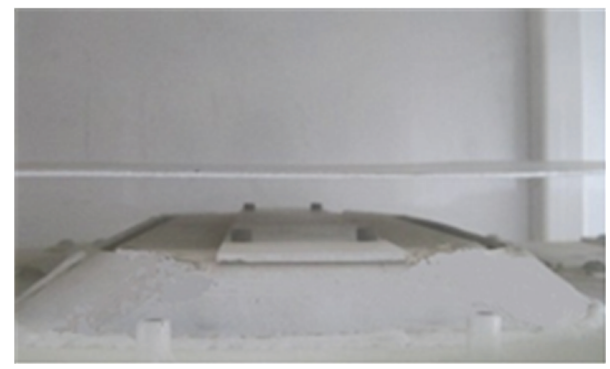

FIGURE 2: The single nozzle and the floating aluminum strip.

to the lower nozzle is adjusted by the blower's rotating speed. Furthermore, the changes of the blower's rotating speed can influence the pressure of the lower nozzle $P_{t 1}$ and that of the upper nozzle $P_{t 2}$.

During the air flotation process, flotation height is an important variable, which is defined as the distance between the rigid web and lower nozzles. If the floating strips are close to the upper or lower nozzles, strip scratch may occur which may cause the product to abandon. Therefore, flotation height should be controlled in a proper position in order to guarantee sufficient margin between the upper nozzles and lower nozzles. After theoretical analysis, the flotation height is determined by various parameters such as air density, density of the aluminum alloy, strip thickness, upper nozzle pressure $P_{t 1}$, and lower nozzle pressure $P_{t 2}$.

2.2. Mechanism Model of Flotation Height. Generally, aluminum strip has strong hardness. When the aluminum strip is floating in the air, the deflection of aluminum strip is small which can be seen in Figure 2. Therefore, in the development of the proposed mechanism model, the aluminum strip shape is considered as straight in the horizontal direction. Based on the above consideration, ground effect theory is applied in this study, which is proven to be useful for describing the aerodynamic characteristics of pressure-pad air bars in air flotation oven $[1,3,5,6]$.

The ground effect theories are worked under the following assumption [3]:

(1) the thickness of jet flow is much smaller than the flotation height $(b / h \ll 1)$ and does not change along the path of the jet;

(2) the flow profile across the jet is uniform;

(3) the jet speed does not change along the path of the jet;
(4) the path of the jet flow has a constant curvature and is tangent to the ground;

(5) the pressure in the region surrounded by the two streams of air jet is constant.

On the basis of ground effect theories, the vertical force balance for the air jet requires

$$
\rho b V_{j}^{2}(1+\cos \theta)=P_{c} * h,
$$

where $\rho$ is the air density, $b$ is the slit nozzle's width, $V_{j}$ is the air velocity, and $P_{c}$ is the cushion pressure (gage pressure). The effective total pressure (gage pressure) of the air jet after the nozzle is

$$
p_{j}=\frac{P_{c}}{2}+\frac{\rho V_{j}^{2}}{2}
$$

where the static pressure is assumed to be the average of the ambient pressure and the cushion pressure, because these two pressures are acting on the two sides of the air jet nozzle. Substituting (1) into (2) the pressure ratio is as follows:

$$
\frac{P_{c}}{P_{t}}=\frac{2(1+\cos \theta)}{h / b+1+\cos \theta}
$$

The lift force per unit length of air bar is

$$
F=P_{c} w+2 \rho b V_{t}^{2} \sin \theta
$$

where $w$ is the distance between the two slot nozzles. The last term in (4) is the momentum change of two air jets in the vertical direction. By eliminating $P_{c}$ and $\rho b V_{t}^{2}$ from (4) using (1) and (3), the equation is as follows:

$$
F=\frac{P_{c}}{P_{t}} *\left(\frac{w}{b}+\frac{h}{b} \frac{2 \sin \theta}{1+\cos \theta}\right) P_{t} * b .
$$

The lower floatation force $F 1$ is

$$
F 1=\frac{P_{c 1}}{P_{t 1}} *\left(\frac{w}{b}+\frac{h}{b} \frac{2 \sin \theta}{1+\cos \theta}\right) n_{1} * P_{t 1} * b,
$$

where $h_{d}$ is the distance between the bottom surface of upper nozzles and the top surface of lower nozzles (seen in Figure 1), and $n_{1}$ is the number of lower nozzles. The upper floatation nozzle's flotation is $h_{d}-h$. The upper nozzles force $F 2$ is

$$
F 2=\frac{P_{c 2}}{P_{t 2}} *\left(\frac{w}{b}+\frac{h_{d}-h}{b} \frac{2 \sin \theta}{1+\cos \theta}\right) n_{2} * P_{t 2} * b,
$$

where $n_{2}$ is the number of upper nozzles. The strip will float at a height where the combination of the aluminum strip's weight and the air force due to the upper nozzle just balances the upward force due to the lower nozzles [5]. Consider

$$
F 1-F 2=G
$$


where $F 1$ is lower floatation force, $F 2$ is upper floatation force, and $G$ is the weight of the strip. Substituting (6) and (7) into (8), (9) is as follows:

$$
\begin{aligned}
& {\left[4 b \sin \theta\left(n_{1} P_{t 1}-n_{2} P_{t 2}\right)-G\right] h^{2}} \\
& \quad+\left[h_{d} G-4 h_{d} b \sin \theta\left(n_{1} P_{t 1}-n_{2} P_{t 2}\right)+2 b(1+\cos ) \theta\right] \\
& \quad \times\left(n_{1} P_{t 1}+n_{2} P_{t 2}\right)(w-2 b) \sin \theta h+b(1+\cos \theta) \\
& \quad \times\left\{h_{d} G+b(1+\cos \theta)\left[G-2 w\left(n_{1} P_{t 1}-n_{2} P_{t 2}\right)\right]\right\} \\
& \quad-2 h_{d} w n_{1} P_{t 1}+4 h_{d} b \sin \theta n_{2} P_{t 2}=0 .
\end{aligned}
$$

The flotation height $h$ can be solved from (9).

\section{Selective Bagging Ensemble Using NCL and GA}

In this study, LSSVR is used as the base learning algorithm. The main contribution of current study focuses on the designing of ensemble method, while LSSVR is directly used without modification. Therefore, LSSVR will not be introduced and the details of it can be founded in $[28,29]$.

In the following, the basic principle of bagging and NCL is firstly introduced. Thereafter, a selective bagging ensemble will be proposed.

3.1. The Basic Idea of Bagging. For bagging algorithm, each training subset contains $n$ learning samples which is drawn randomly with replacement from the original training set of size $N$. Such a training subset is called a bootstrap replicate of the original set. Instead of making predictions from a single model that is fitted to the observed data, a number of predictions models are developed to predict the relationship between input and output variables. Each model is developed from the multiple models which are combined to improve model accuracy and robustness [21, 22].

Let $T=\left\{\left(x_{p}, y_{p}, p=1, \ldots, N\right)\right\}$ denote a regression type training set, and the SVM algorithm uses $T$ to construct a regression predictor $F_{R}(x, T)$ to predict $y$ values. Let $F$ be a bagging ensemble algorithm obtained as a simple averaging combination of $M$ predictors; that is,

$$
F\left(x_{p}\right)=\frac{1}{M} \sum_{i=1}^{M} f_{i}\left(x_{p}\right)
$$

where $M$ is the number of the individual SVM in the ensemble algorithm, $f_{i}\left(x_{p}\right)$ is the output of SVM $i$ on the data set, and $F\left(x_{p}\right)$ is the output of the ensemble algorithm on the data set.

3.2. The Basic Idea of NCL. NCL implicitly creates different training sets by encouraging different individual models to learn different parts or aspects of the training data, so that all networks can be trained simultaneously and interactively on the same training data set $T[29,30]$ as follows:

$$
\begin{aligned}
C_{i}= & \left(f_{i}\left(x_{p}\right)-F\left(x_{p}\right)\right) \\
& \times \sum_{j \neq 1}\left(f_{i}\left(x_{p}\right)-F\left(x_{p}\right)\right)=-\left(f_{i}\left(x_{p}\right)-F\left(x_{p}\right)\right)^{2} .
\end{aligned}
$$

The error function $E_{i}$ for SVR $i$ in negative correlation learning is defined as

$$
\widehat{E}_{i}=\sum_{p=1}^{N}\left(\frac{1}{2}\left(f_{i}\left(x_{p}\right)-F\left(x_{p}\right)^{2}+\lambda C_{i}\right)\right) .
$$

The parameter $0 \leq \lambda \leq 1$ is used to adjust the strength of the penalty. The simple averaging of the ensemble in negative correlation learning is defined as:

$$
\widehat{E}_{i}=\frac{1}{M} \sum_{i=1}^{M} \sum_{p=1}^{N}\left(\frac{1}{2}\left(f_{i}\left(x_{p}\right)-F\left(x_{p}\right)^{2}+\lambda C_{i}\right)\right) .
$$

3.3. Selective Bagging Using NCL. In bagging ensemble algorithm, the accuracy of the individual model is not well controlled. If there are some uncorrected individual models with large bias, the overall prediction performance of the ensemble model may deteriorate. Therefore, the accuracy of the individual model is managed in this paper. The individual model with undesirable precision is retrained until its desired accuracy is obtained.

Moreover, the individual models in the original bagging ensemble are trained independently. There is insufficient cooperation between them, which may worse the overall prediction performance. Furthermore, the original bagging ensemble is inefficient due to its relatively large ensemble size. In order to address above two problems, NCL algorithm is introduced to bagging ensemble algorithm, so the cooperation between individual models can be improved. By virtue of NCL, the individual models cooperated with each other in the original bagging ensemble and the redundant individual models with no contribution to prediction accuracy can be pruned from original bagging ensemble.

By modification of NCL, the error function $E_{i}$ for $i$ th individual model in selective bagging is as follows:

$$
\widehat{E}_{i}=w_{i} \cdot \sum_{p=1}^{N}\left(\frac{1}{2}\left(f_{i}\left(x_{p}\right)-F\left(x_{p}\right)^{2}+\lambda C_{i}\right)\right) .
$$

Similar to NCL, the parameter $0 \leq \lambda \leq 1$ is used to adjust the strength of the penalty. The simple average of selective bagging is shown as follows:

$$
\widehat{E}=\sum_{i=1}^{M} w_{i} \sum_{p=1}^{N}\left(\frac{1}{2}\left(f_{i}\left(x_{p}\right)-F\left(x_{p}\right)^{2}+\lambda C_{i}\right)\right),
$$

subject to $\sum_{i} w_{i}=1, \quad 0 \leq w_{i} \leq 0.5, i=2,3, \ldots, M$.

Then, the remaining problem is the determination of the weight $w_{i}$, which will be described in the following section. 


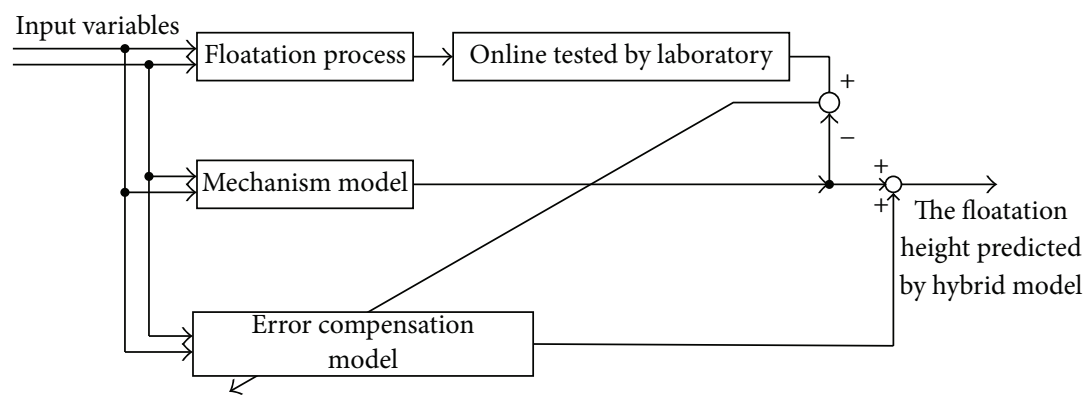

FIgURE 3: Hybrid model structure.

3.4. Solving the Selective Bagging Ensemble Problem Using Genetic Algorithm. In our proposed algorithm, genetic algorithm is used to solve the optimization problem (15). The optimal subset is selected from the pool of the ensemble algorithms.

In genetic algorithm, the chromosomes are represented by a binary string of length $K \times n$ in which $K$ is fixed in algorithm main. Individual algorithm is encoded in a binary length of size $n$. To illustrate this point, the individual SVR algorithms are encoded as follows: (in $1=0000$, in $2=0001$, $\ldots$, in $_{\mathrm{GA}_{p}}=1111$ ) and the chromosomes are encoded as follows: $\left(\mathrm{ch} 1=00000000, \mathrm{ch} 2=001011, \ldots, \mathrm{ch}_{\mathrm{GA}_{p}}=111001\right)$.

In the chromosomes of existing population, individuals are repeatedly selected for breeding until the new population is saturated. The fitness proportionate selection of roulettewheel is applied to this proposed algorithm, and the fitness function is (15). The chromosomes will be selected, crossed over and mutated in the optimal process. In the selecting process, the standard elitism approach is adopted, so the evolution process can become more stable and converges earlier. During the crossover and mutation processes, the singlepoint crossover method and flip-flop single-point mutation is applied. Generally, the average fitness function will be improved according to the genetic operators of crossover and mutation. However, undesired chromosomes may appear repeatedly in the process. For example, in the two chromosomes $(110100,100110), n=3$ and $K=2$, if single point crossover point occurs, the new spring will be (110110, 100100). Similarly, given a chromosome 111101, if mutation happens at the second point, the new offspring will be 111111. Under such circumstances, the chromosomes are treated as bad individuals for evolution.

In our method, the optimization problem (15) is solved in a sequential way. The ensemble size increases progressively and the ensemble size is finally confined by a simple approach. Specifically, genetic algorithm is firstly used to solve the optimization problem (15) with a fixed ensemble size 2 . Therefore, it can select two individual models from a pool of individual models to compose an ensemble model $E_{2}$. Similarly, an ensemble model $E_{3}$ with three individual models can then be established. Thereafter, comparison is carried out between $E_{2}$ and $E_{3}$. If the value of objective function of $E_{3}$ is smaller than $E_{2}$, the algorithm is expanded to find the best ensemble with four individual models and so on. The algorithm converges and the increment of ensemble size stops when the value of objective function corresponding to the ensemble model increases. It can be concluded that the minimum ensemble error and the optimal ensemble size can be obtained by this ensemble way. In each iteration, it can be found that genetic algorithm (GA) is used to search for the best ensemble size $K$ that minimizes the fitness function (15). The selection process is explained in main algorithm.

The hybrid selective ensemble process is as follows.

\section{Algorithm Main}

Step 1. Generate training subset $T_{i}$ from $T$ by bootstrap sampling algorithm. Train an individual model $f_{i}$ on the training subset $T_{i}$ by SVM algorithm.

Step 2. Calculate the training error $E_{i}$ of $f_{i}$ by (14). If $E_{i}>e_{1}$ repeat Step 1; otherwise if $i=i+1$, build SVM model $f_{i}$ with $T_{i}$.

Step 3. $K=2$.

Step 4. Find the best ensemble individual models of $K$ and $K+1$ models by GA.

Step 5. If the $f_{i}$ is selected in Step 4 , then $w_{i}=1 / K$; otherwise, $w_{i}=0$.

Step 6. Evaluate ensemble error $\mathrm{En}_{K}$ and $\mathrm{En}_{K+1}$ on validation set $D_{v}$ by (15). Evaluate $\mathrm{En}_{K+1}$ on $D_{v}$.

Step 7. If $\operatorname{En}_{K}>\operatorname{En}_{K+1}$ and $K=K+1$, repeat Step 4; otherwise go to Step 8.

Step 8. Output: $F\left(x_{p}\right)=(1 / K) \sum_{i=1}^{K} f_{i}\left(x_{p}\right)$.

\section{Hybrid Model Based on Selective Bagging Ensemble Method}

In this paper, a hybrid flotation height prediction model is developed which is made up of mechanism model and data model. The data model is a new method based on selective bagging ensemble, and it is used as the error compensation model of hybrid model. The structure of the hybrid model is shown in Figure 3. Based on the mechanism analysis, the input variables of the hybrid model are determined: air 


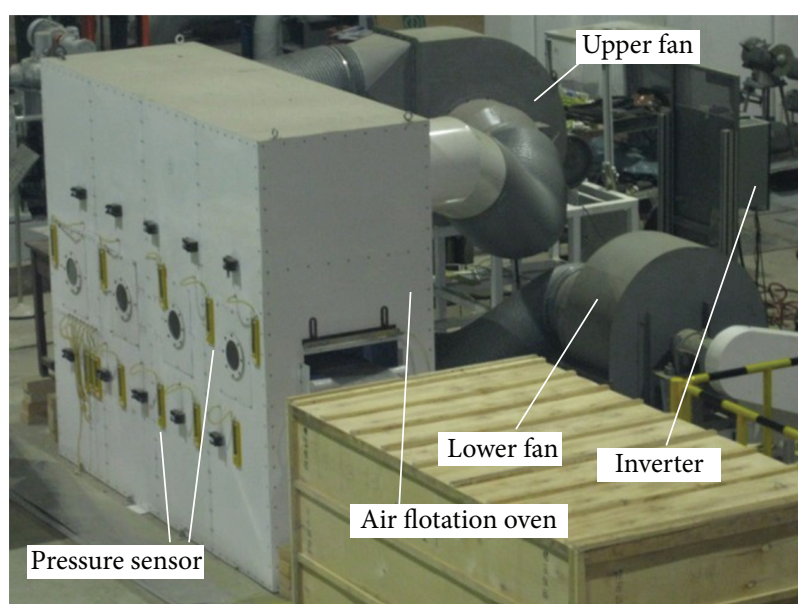

FIGURE 4: The diagram of air flotation system.

density, the upper nozzle's pressure, the lower nozzle's pressure, the strip thickness, and aluminum strip density. The flotation height in air flotation oven is predicted based on the hybrid model.

In the hybrid model, the error compensation model is used to compensate the flotation height modeling error of mechanism model. As a result, the prediction error of mechanism model can be well compensated.

\section{Practical Application and Experiments}

The proposed flotation height prediction model is validated on an experimental equipment which is located in The State Key laboratory of Rolling and Automation in Northeastern University. A set of experimental data collected from this experimental air flotation oven. The experimental air flotation oven can be seen in Figure 4.

From Figure 4, the air flotation oven system consists of lower fan, upper fan, two inverters, pressure sensor, and so forth. The size of the air flotation system is $3 \times 3 \times 2.2 \mathrm{~m}$. The inverter is SIEMENS MM440. The pressure sensor is U-tube whose range is $0-2000 \mathrm{~Pa}$ and resolution is $10 \mathrm{~Pa}$. The upper nozzle pressure and lower nozzle pressure are measured by Utubes. A Leica hand-hold distance finder is used to measure the flotation height. The range and resolution of hand-hold distance finder are $50 \mathrm{~m}$ and $1 \mathrm{~mm}$, respectively. The handhold distance finder is just used for convenient measurement under experimental conditions and it does not exist in industry process.

The inner flow guide structure of air flotation oven is shown in Figure 5. The air flotation oven consists of upper air container, lower air container, upper nozzles, lower nozzles, and so forth.

In air flotation oven, the speeds of upper and lower fan are, respectively, controlled by a variable-frequency inverter, respectively, (AC drive). The air successively flows through fans, air containers and nozzles and finally is ejected to the surface of aluminum strip.

The upper and lower jet speeds are adjusted by fans. Aluminum strip floats on a fixed height under different speed

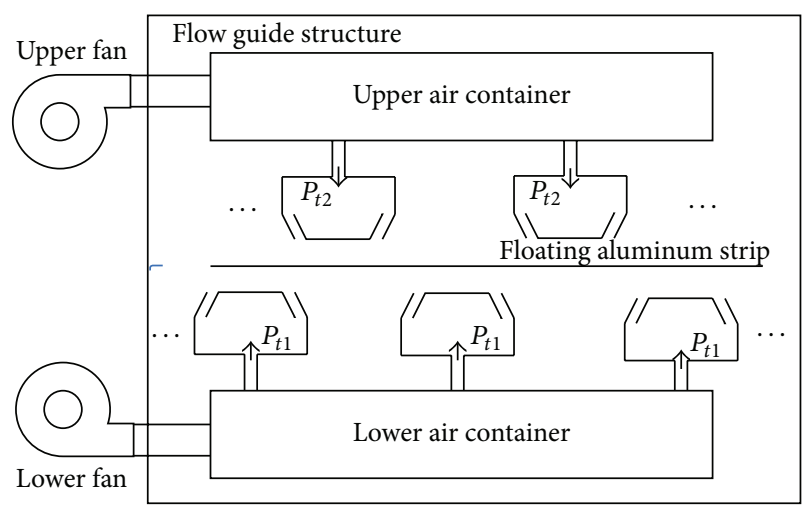

FIgURE 5: The inner flow guide structure of air flotation device.

ratio of upper fan and lower fan. Under experimental conditions, the ranges of jet speeds and mach numbers are 0$25 \mathrm{~m} / \mathrm{s}$ and $0-0.0735$ (incompressible fluid). The mechanical structure let fluid flow along the length direction of experiment equipment, so two-dimensional air flow is considered.

In our experiment, the width of aluminum strip is $300 \mathrm{~mm}$. The thickness of the strip varies from $0.4 \mathrm{~mm}$ to $2 \mathrm{~mm}$ at the interval of $0.2 \mathrm{~mm}$. Finally, 850 samples that cover various working conditions are collected for training the hybrid model. Additionally, 32 samples that specified to 4 given working conditions are collected for testing the hybrid model and process analysis.

In practical experiment, there is measuring error in process data, because of the instrumentation precision and interfering signal. With the deviation of process data, the accuracy of the model will be degraded. The abnormal data should be eliminated. The statistics discriminant method of Pauta criteria is applied in the experiment. The principle of Pauta is as follows: sample data set is $X=\left\{x_{1}, x_{2}, \ldots, x_{n}\right\}$ and $\bar{X}$ is the average value. Deviation value is $v_{i}=x_{i}-\bar{X}(i=1,2, \ldots, n)$. The standard deviation is calculated according to Bayesian formula as follows:

$$
\sigma=\sqrt{\sum_{i=1}^{n}\left[\frac{v_{i}^{2}}{(n-1)}\right]} .
$$

If the sample data $x_{i}^{\prime}$ deviation value is $\left|v_{i}\right| \geq 3 \sigma$, the sample data should be eliminated.

Comprising the hybrid selective bagging ensemble model (SBEH), single SVM hybrid model (SVMH), basic bagging hybrid model (BBH), and mechanism model (MM) are also applied to this experiment. There are eight parameters that should be tuned in our selective ensemble method SEH $\left(\mathrm{GA}_{g}\right.$, $\mathrm{GA}_{p}, \mathrm{GA}_{c}, \mathrm{GA}_{m}$, base learner size, individual error limit value $e_{1}$, and $\left.\lambda\right)$, three parameters for $\mathrm{BBH}\left(\sigma^{2}\right.$ and $\gamma$ for base learner, and ensemble size), two parameters for SVMH $\left(\sigma^{2}\right.$ and $\gamma$ ). The base learner size of our proposed method and $\mathrm{BBH}$ ensemble have been set to 128 .

In $\mathrm{SBEH}$ model, maximum number of generations $\mathrm{GA}_{g}$, population size $\mathrm{GA}_{p}$, crossover rate $\mathrm{GA}_{c}$, the mutation rate $\mathrm{GA}_{m}$ of the genetic algorithm, individual error control $e_{1}$, and the penalty parameter $\lambda$ have been fixed to $200,128,0.75,0.02$, 


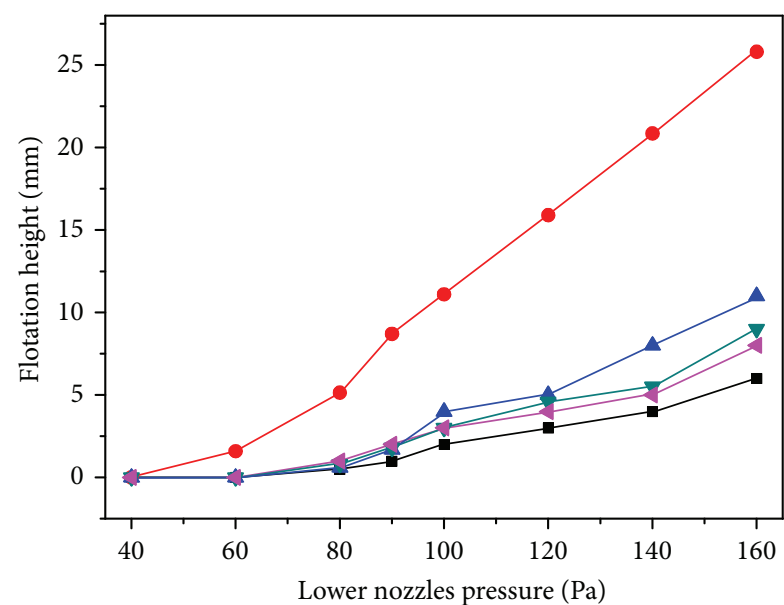

$\rightarrow$ BBH's prediction value $\rightarrow$ SBEH's prediction value

$\rightarrow$ MM's prediction value $\leftarrow$ Actual value

$\rightarrow$ SVRH's prediction value

(a) Thickness $=1 \mathrm{~mm}$, upper pressure $=0 \mathrm{~Pa}$

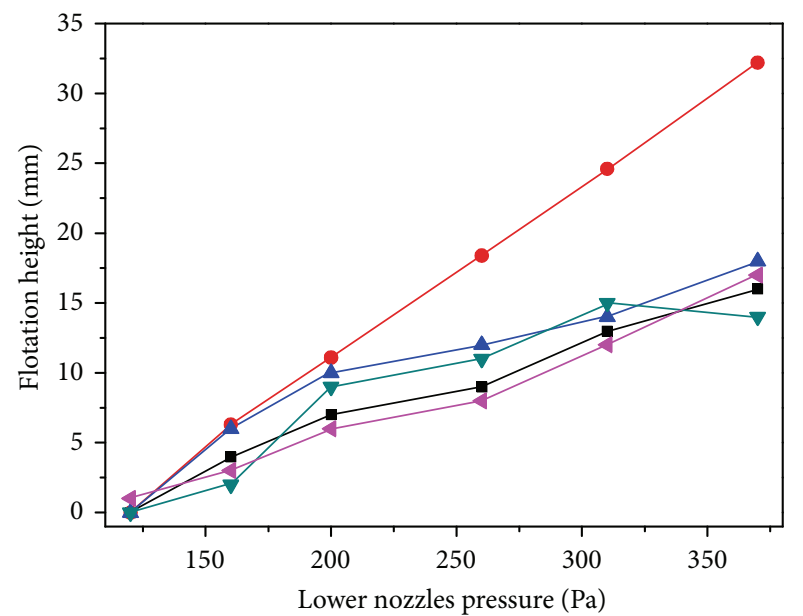

$\rightarrow$ SBEH's prediction value $\rightarrow$ SVRH's prediction value

$\rightarrow$ MM's prediction value $\multimap$ Actual value

$\_$BBH's prediction value

(c) Thickness $=2 \mathrm{~mm}$, upper pressure $=0 \mathrm{~Pa}$

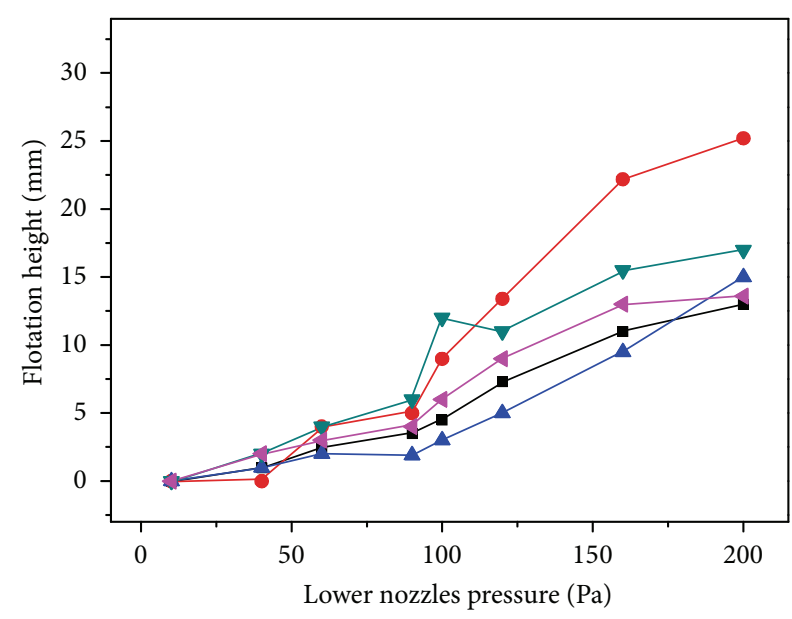

$\rightarrow$ SBEH's prediction value $\rightarrow$ SVRH's prediction value

$\rightarrow$ MM's prediction value $\longleftarrow$ Actual value

$\leftarrow$ BBH's prediction value

(b) Thickness $=1 \mathrm{~mm}$, upper pressure $=65 \mathrm{~Pa}$

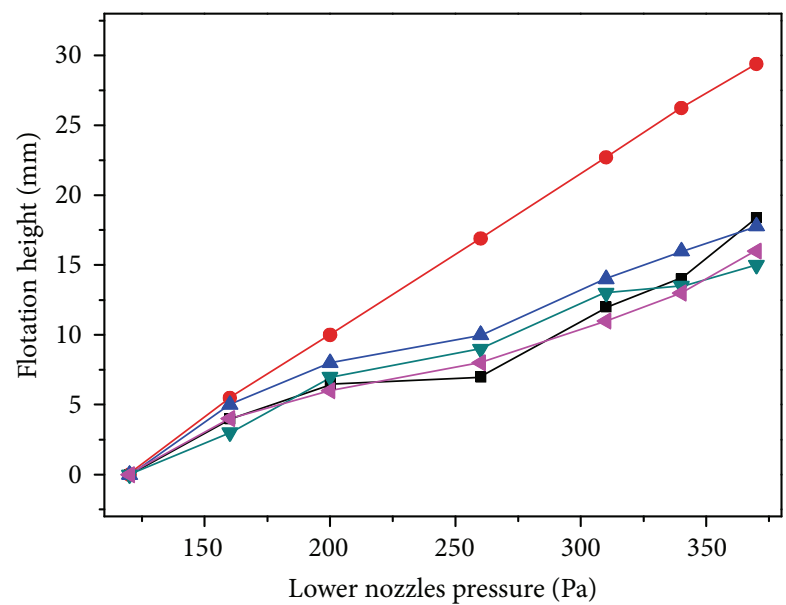

$\rightarrow$ SBEH's prediction value $\rightarrow$ BBH's prediction value

$\rightarrow$ MM's prediction value $\leftarrow$ Actual value

$\simeq$ SVRH's prediction value

(d) Thickness $=2 \mathrm{~mm}$, upper pressure $=65 \mathrm{~Pa}$

FIGURE 6: The prediction and actual flotation height under different work condition.

10 , and 0.4 , respectively. The bootstrap sampling size of $\mathrm{BBH}$ have been fixed to roughly $60 \%$ of the training set. Parameters $\sigma^{2}$ and $\gamma$ of single SVM hybrid model and BBH model has been fixed to 4 and $2^{12}$.

Different information about the predictive capacity of the model can be measured through RMSE and MAE. The RMSE and MAE are defined as

$$
\begin{aligned}
\text { RMSE } & =\sqrt{\frac{1}{N} \sum_{i=1}^{N}\left(Y_{i_{\text {observed }}}-Y_{i_{\text {estimate }}}\right)^{2},} \\
\text { MAE } & =\frac{1}{N} \sum_{i=1}^{N}\left|Y_{i_{\text {observed }}}-Y_{i_{\text {estimate }}}\right|
\end{aligned}
$$

where $N$ is the number of individual models and $Y_{i}$ is the flotation height.

The experiment is carried on an experimental air flotation oven. In Figure 6, thickness of the aluminum strip is $1 \mathrm{~mm}$, and $2 \mathrm{~mm}$. The width of the aluminum strip is $300 \mathrm{~mm}$. The flotation height is tested under different lower nozzle pressure $P_{t 1}$ and upper nozzle pressure $P_{t 2}$. The prediction value and actual value of flotation height are shown in Figure 6.

Figure 6 shows the predicted flotation height based on the proposed hybrid selective bagging ensemble method. Hybrid selective bagging ensemble model has better generalization ability and is precise than other models. Table 1 shows RMSE and MAE of SBEH, SVMH, MM, and BBH model.

From Table 1, it can be concluded that our proposed approach SBEH outperforms the other three algorithms. 
TABLE 1: Comparison of four models.

\begin{tabular}{lcc}
\hline Model & RMSE & MAE \\
\hline MM & 9.07 & 7.57963 \\
BBH & 2.0503 & 1.559259 \\
SVMH & 2.3868 & 1.988889 \\
SBEH & 1.195385 & 0.903704 \\
\hline
\end{tabular}

According to the above experimental results, the application of hybrid method SBEH can bring improvements as shown in Figure 5 and Table 1. These results reveal that hybrid ensemble method is the more dominated than the mechanism algorithm. The possible reason may be that fluid interaction that exists between different nozzles and the fluid interaction is not well described by mechanism model while the hybrid ensemble method is able to learn the fluid interaction process by its data model part that possesses good self-learning ability. The other possible reason may be that these assumptions in mechanism model and other unknown factors in process are learned by date model part of hybrid ensemble method. Furthermore, it can be found from the comparison result that the proposed hybrid model outperforms the single SVR hybrid model and basic bagging hybrid model. Therefore, it can be concluded that the proposed hybrid method is able to further improve the prediction performance in the prediction of flotation height.

\section{Conclusions}

In this paper, a mathematical mechanism model of flotation height in air flotation oven is firstly developed. Thereafter, a hybrid model is designed by the proposed selective bagging ensemble method. This proposed model can compensate the error of the mechanism model. The proposed hybrid model can combine the well generalization performance of mechanism modeling method and the excellent learning ability of machine learning algorithms. Thereby better flotation height prediction performance can be obtained. The simulation results show that the proposed hybrid selective bagging ensemble model does consistently improve the predicted precision versus MM model, BBH model, and SVMH model for flotation height. In summary, the proposed hybrid modeling algorithm has a good potential in the actual air flotation oven.

\section{Acknowledgments}

The authors gratefully acknowledge supports provided by Science and Technology Projects in Liaoning Province L2013113 and the Fundamental Research Funds for the Central Universities of China under Project N110604011.

\section{References}

[1] H.-K. Cho, Flow-Induced Vibration of A Web Floating Over a Pressure-Pad Air Bar, Oklahoma State University, 2005.

[2] H. Cho, An Alytical and Computational Study of the Asymmetry of Webs Pass Gover, Konkuk University, 1997.
[3] M. J. Davies and D. H. Wood, "The basic aerodynamics of flotation," Journal of Fluids Engineering, Transactions of the ASME, vol. 105, no. 3, pp. 323-328, 1983.

[4] Y. B. Chang, R. P. Swanson, and P. M. Moretti, "Resiliency of an air-floated web," in Proceedings of the 5th International Conference on Web Handling, pp. 543-558, 1999.

[5] Y. B. Chang, R. P. Swanson, and P. M. Moretti, "Longitudinal and out-of-plane stiffness of a web in an air-flotation oven," in Proceedings of the ASME International Mechanical Engineering Congress and Exposition (IMECE '99), pp. 435-443, November 1999.

[6] P. M. Moretti, "Lateral deflections of Webs in air-flotation ovens," Journal of Applied Mechanics, Transactions ASME, vol. 71, no. 3, pp. 314-320, 2004.

[7] H. Cho, Pressure-Pad Air Bars, Oklahoma State University, 1999.

[8] M. Wu and Y. Zhao, "Knowledge-based approach to assembly sequence planning for wind-driven generator," Mathematical Problems in Engineering, vol. 2013, Article ID 908316, 7 pages, 2013.

[9] M. Štěpnička, P. Cortez, J. Peralta Donate, and L. Štěpničková, "Forecasting seasonal time series with computational intelligence: on recent methods and the potential of their combinations," Expert Systems with Applications, vol. 40, no. 6, pp. 19811992, 2013.

[10] Y.-J. Lee and S.-Y. Huang, "Reduced support vector machines: a statistical theory," IEEE Transactions on Neural Networks, vol. 18, no. 1, pp. 1-13, 2007.

[11] C. Chen, J. Xia, J. Liu, and G. Feng, "Nonlinear inversion of potential-field data using a hybrid-encoding genetic algorithm," Computers \& Geosciences, vol. 32, no. 2, pp. 230-239, 2006.

[12] S.-Z. Gao, J.-S. Wang, and N. Zhao, "Fault diagnosis method of polymerization Kettle equipment based on rough sets and BP neural network," Mathematical Problems in Engineering, vol. 2013, Article ID 768018, 12 pages, 2013.

[13] R. Stoean and C. Stoean, "Modeling medical decision making by support vector machines, explaining by rules of evolutionary algorithms with feature selection," Expert Systems with Applications, vol. 40, no. 7, pp. 2677-2686, 2013.

[14] D. Wang and M. Alhamdoosh, "Evolutionary extreme learning machine ensembles with size control," Neurocomputing, vol. 102, pp. 98-110, 2013.

[15] S.-W. Fei and Y. Sun, "Forecasting dissolved gases content in power transformer oil based on support vector machine with genetic algorithm," Electric Power Systems Research, vol. 78, no. 3, pp. 507-514, 2008.

[16] W. Chu and Z. Ghahramani, "Gaussian processes for ordinal regression," Journal of Machine Learning Research, vol. 6, pp. 1019-1041, 2005.

[17] M. C. Kennedy and A. O'Hagan, "Bayesian calibration of computer models," Journal of the Royal Statistical Society B, vol. 63, no. 3, pp. 425-464, 2001.

[18] C. E. Rasmussen and C. K. I. Williams, Gaussian Processes for Machine Learning, Adaptive Computation and Machine Learning, MIT Press, Cambridge, Mass, USA, 2006.

[19] D. Hernández-Lobato, G. Martínez-Muñoz, and A. Suárez, "Empirical analysis and evaluation of approximate techniques for pruning regression bagging ensembles," Neurocomputing, vol. 74, no. 12-13, pp. 2250-2264, 2011.

[20] Z.-H. Zhou, J. Wu, and W. Tang, "Ensembling neural networks: many could be better than all," Artificial Intelligence, vol. 137, no. 1-2, pp. 239-263, 2002. 
[21] T. Chen and J. Ren, "Bagging for Gaussian process regression," Neurocomputing, vol. 72, no. 7-9, pp. 1605-1610, 2009.

[22] K. Wang, T. Chen, and R. Lau, "Bagging for robust non-linear multivariate calibration of spectroscopy," Chemometrics and Intelligent Laboratory Systems, vol. 105, no. 1, pp. 1-6, 2011.

[23] L. Breiman, "Bagging predictors," Machine Learning, vol. 24, no. 2, pp. 123-140, 1996.

[24] M. Behzad, K. Asghari, M. Eazi, and M. Palhang, "Generalization performance ofsupport vector machines and neural networks in runoff modeling," Expert Systems with Applications, vol. 36, no. 4, pp. 7624-7629, 2009.

[25] X. Yao and Y. Liu, "Making use of population information in evolutionary artificial neural networks," IEEE Transactions on Systems, Man, and Cybernetics B, vol. 28, no. 3, pp. 417-425, 1998.

[26] Y. Liu and X. Yao, "Simultaneous training of negatively correlated neural networks in an ensemble," IEEE Transactions on Systems, Man, and Cybernetics B, vol. 29, no. 6, pp. 716-725, 1999.

[27] S. Masoudnia, R. Ebrahimpour, and S. A. A. Abbaszadeh Aranib, "Combining features of negative correlation learning with mixture of experts in proposed ensemble methods," Applied Soft Computing, vol. 12, no. 11, pp. 3539-3551, 2012.

[28] S. Ismail, A. Shabri, and R. Samsudin, "A hybrid model of self-organizing maps (SOM) and least square support vector machine (LSSVM) for time-series forecasting," Expert Systems with Applications, vol. 38, no. 8, pp. 10574-10578, 2011.

[29] S. Li and L.-K. Dai, "Classification of gasoline brand and origin by Raman spectroscopy and a novel R-weighted LSSVM algorithm," Fuel, vol. 96, pp. 146-152, 2012.

[30] G. Hu, Z. Mao, D. He, and F. Yang, "Hybrid modeling for the prediction of leaching rate in leaching process based on negative correlation learning bagging ensemble algorithm," Computers and Chemical Engineering, vol. 35, no. 12, pp. 2611-2617, 2011. 


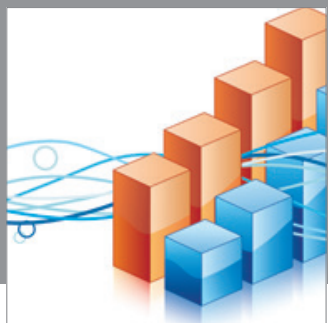

Advances in

Operations Research

mansans

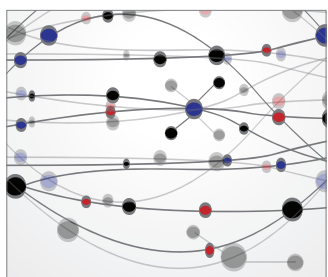

The Scientific World Journal
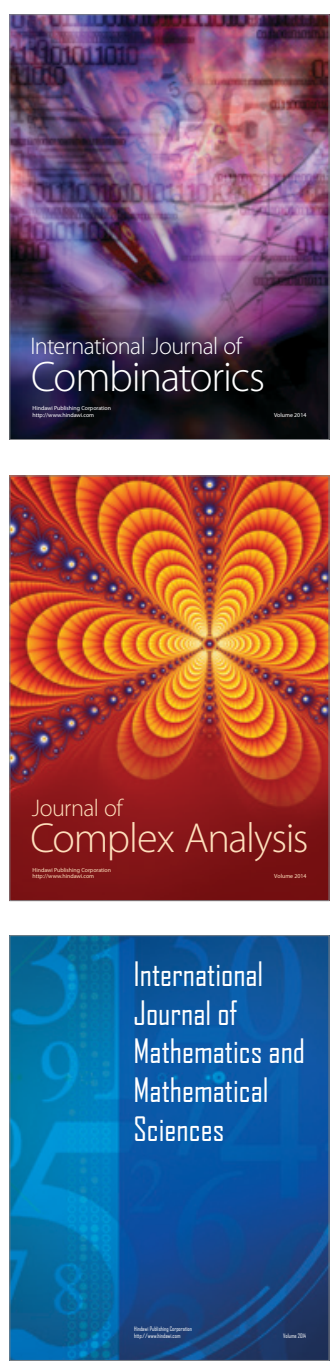
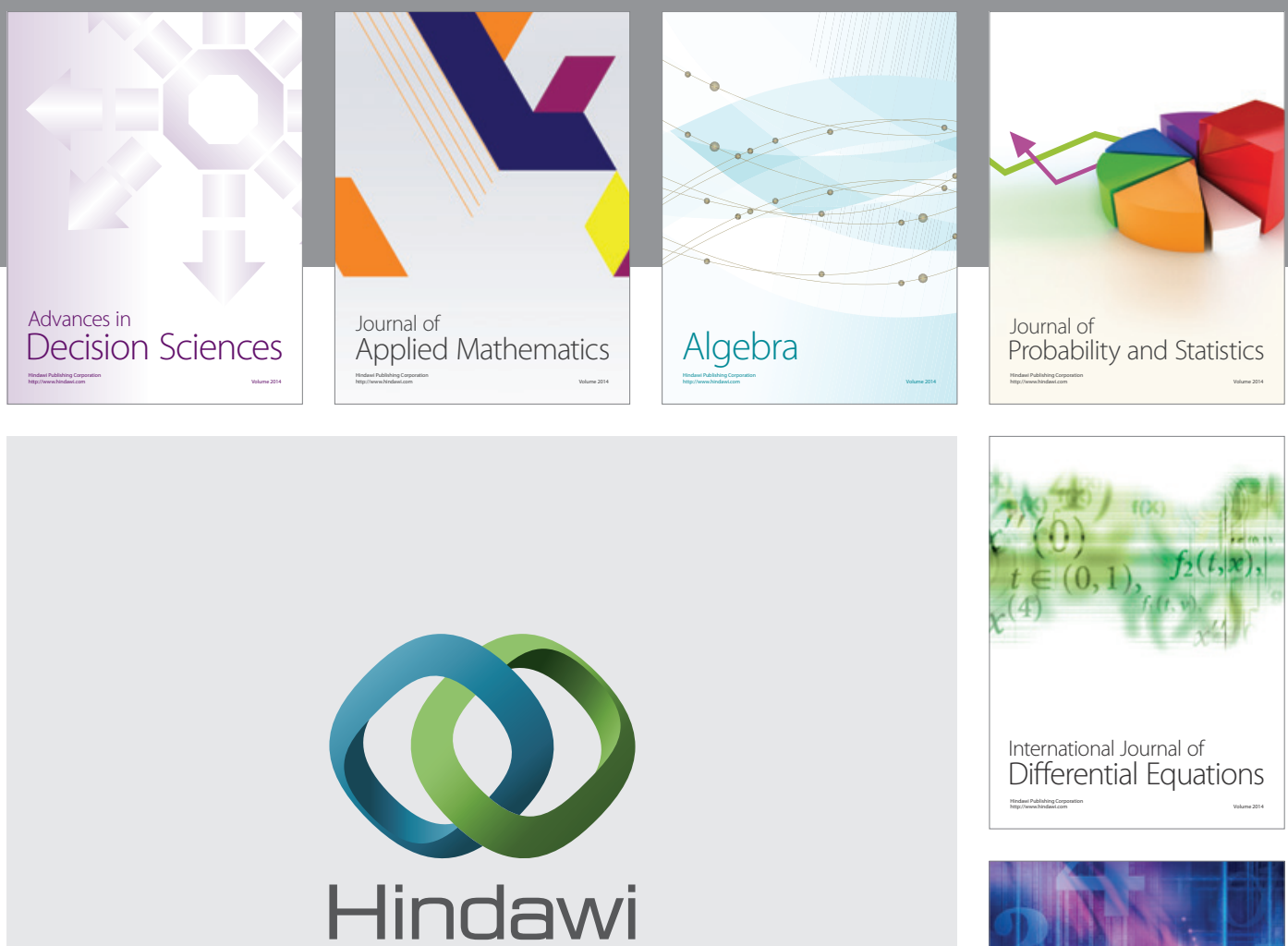

Submit your manuscripts at http://www.hindawi.com
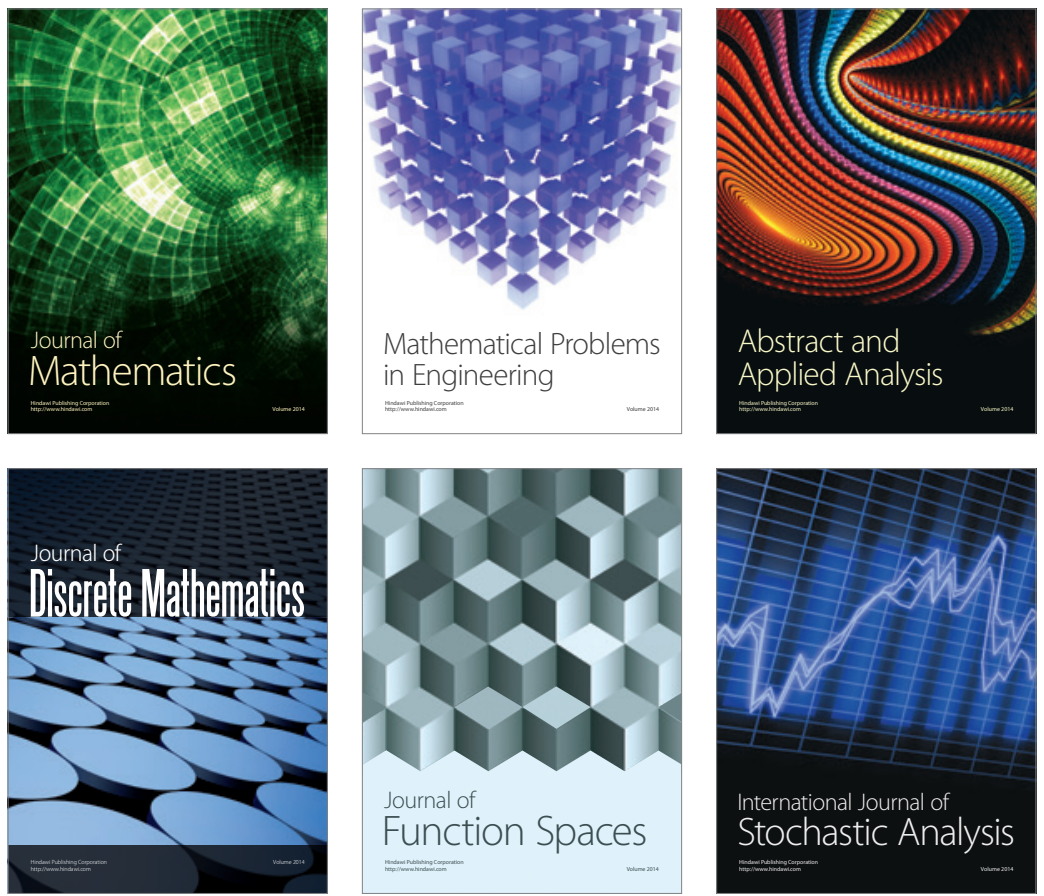

Journal of

Function Spaces

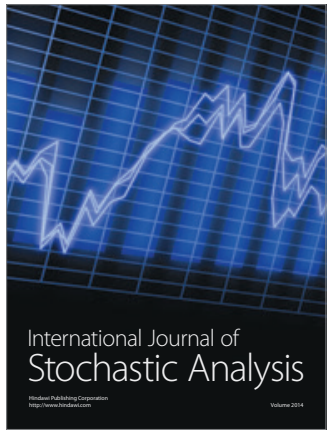

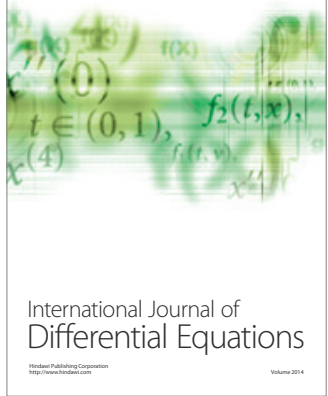
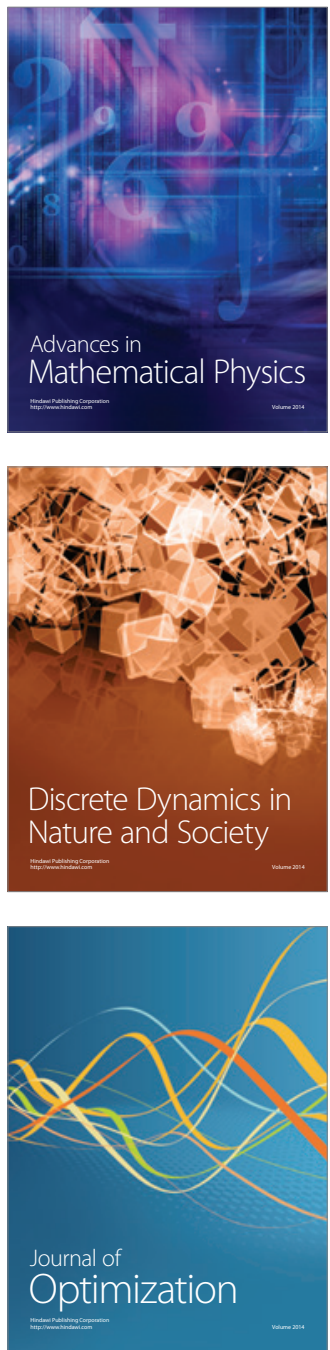